FACTA UNIVERSITATIS

Series: Mechanical Engineering Vol. 17, No 2, 2019, pp. 191 - 205

https://doi.org/10.22190/FUME190215025C

Original scientific paper

\title{
SOME SIMPLE RESULTS ON THE MULTISCALE VISCOELASTIC FRICTION
}

\section{Michele Ciavarella, Antonio Papangelo}

Politecnico di BARI, Center of Excellence in Computational Mechanics, Bari, Italy

\begin{abstract}
The coefficient of friction due to bulk viscoelastic losses corresponding to multiscale roughness can be computed with Persson's theory. In the search for a more complete understanding of the parametric dependence of the friction coefficient, we show asymptotic results at low or large speed for a generalized Maxwell viscoelastic material, or for a material showing power law storage and loss factors at low frequencies. The ascending branch of friction coefficient at low speeds highly depends on the rms slope of the surface roughness (and hence on the large wave vector cutoff), and on the ratio of imaginary and absolute value of the modulus at the corresponding frequency, as noticed earlier by Popov. However, the precise multiplicative coefficient in this simplified equation depends in general on the form of the viscoelastic modulus. Vice versa, the descending (unstable) branch at high speed mainly on the amplitude of roughness, and this has apparently not been noticed before. Hence, for very broad spectrum of roughness, friction would remain high for quite few decades in sliding velocity. Unfortunately, friction coefficient does not depend on viscoelastic losses only, and moreover there are great uncertainties in the choice of the large wave vector cutoff, which affect friction coefficient by orders of magnitudes, so at present these theories do not have much predictive capability.
\end{abstract}

Key Words: Roughness, Contact Mechanics, Rubber Friction, Persson's Theories, Adhesion

\section{INTRODUCTION}

In contact mechanics and tribology, roughness plays a fundamental role for adhesion, friction, lubrication, sealing, despite it is very difficult to make any quantitative predictions depending on it. In particular, in elastic contact fractal roughness leads to a "ill-posed"

Received February 15, 2019 / Accepted May 25, 2019

Corresponding author: Michele Ciavarella

Politecnico di BARI, Center of Excellence in Computational Mechanics, Viale Gentile 182, 70126 Bari, Italy

E-mail: Mciava@poliba.it 
solution (Ciavarella et al., [1], Persson [2]), in which the "real area of contact" and many (but not all) other physical quantities, cannot be precisely defined because they strongly depend on the tail of the power spectrum of roughness (Persson et al., [3]) and in particular on where this tail is truncated. The classical asperity models like Greenwood \& Williamson [4] initially did not recognize this problem, and anyway solve less accurately the mathematical problem of elastic rough contact, where Persson's theory has today elucidated many aspects (see Putignano et al., [5]). The original Persson's theory was aimed at a very practical problem with many scientific and technological applications, estimating friction due to viscoelastic losses in sliding of viscoelastic bodies on hard substrates. However, it seems that most often the pure viscoelastic contribution of friction is not sufficient to explain some experimental results, and possible other mechanisms like adhesion are also put forward (Lorenz et al., [6]). This perhaps somehow limits the interest in obtaining exact results on the viscoelastic losses, and anyway suggests simple formulations should be preferred given the uncertainties on the considerable number of arbitrary parameters which need to be estimated anyway.

The spectrum of roughness is often assumed to be of power law form although is not precisely defined neither at very low wave vectors, nor at high wave vectors, due to limitations in measuring instruments, and the need to use various instruments to obtain a measurement over a very broad range of scales. In particular, the truncation of the spectrum of the surface will critically affect some results. For example, Lorenz et al. [6] suggest that (perhaps in typical present tyre-road contacts?) the truncation wave vector should occur where the rms slope reaches

$$
h_{r m s}^{\prime}\left(q_{1}\right)=1.3 \text {, }
$$

although we have found no data to interpret the motivation and hence substantiate the generality of this recommendation, and no independent researcher has suggested alternative criteria. Other authors (Carbone \& Putignano [7]) suggest many factors could be associated to the truncation cutoff, including small dirt particles or rubber wear particles, but do not give precise suggestions. Hence, while the mathematical problem is relatively easy to formulate, and the multiscale nature of roughness is postulated to be of critical importance, the actual practical solution of this crucial point is left somehow more obscure.

Even less discussed is the other truncation in the spectrum, that at low wave vectors, despite being clear that by enlarging the size of the specimen it is very likely that some power content appears - and anyway, one could argue that even shape or undulations of the pavement could occur and may, in principle, play a role for friction, as we shall see more in details where in particular, in the following.

The theory by Persson [2] is based on exact solution for sliding of a rough rigid random surface in full contact with a viscoelastic medium. The exact result for the friction coefficient would be

$$
\mu=\frac{1}{2} \int_{q_{0}}^{q_{1}} \mathrm{~d} q q^{3} U(q) \times \int_{0}^{2 \pi} \mathrm{d} \phi \cos (\phi) \operatorname{Im} \frac{E(q v \cos (\phi))}{\left(1-v^{2}\right) \sigma_{0}},
$$

where $\sigma_{0}$ is the nominal contact stress, $U(q)$ the surface displacements power spectrum (defined as a function of wave vector $q$ ), where $q_{0}$ is the smallest (relevant) roughness wave vector. Also, $E(\omega)$ is the complex modulus of the viscoelastic material. 
However, since full contact is a very remote condition in practical applications, the real condition of partial contact is solved by making various clever approximations:

1) The PSD (power spectrum density) of displacements, $U(q)$, which would be strictly required to make a correct theory, is approximated with the PSD of the roughness $C(q)$

$$
u_{z}(\mathbf{x}) \simeq h(\mathbf{x}) \text { or } U(q) \simeq C(q)
$$

Since power loss occurs mainly in the contact area, and since it is known that the two PSDs are parallel curves, the error can be fixed a posteriori with corrective factors.

2) The power loss integration is weighted by function $P(q)=A(\zeta) / A_{0}$ which is the relative contact area when the interface is observed at magnification $\zeta=q / q_{0}$, where $A_{0}$ is the nominal contact area. On the grounds that only the portion of the area which is actually in contact really forces the surface to deform and undergo the viscoelastic deformation

$$
\begin{gathered}
P(q)=A(\zeta) / A_{0}=\operatorname{erf}\left(\frac{1}{2 \sqrt{G}}\right), \\
G=\frac{1}{8} \int_{q_{0}}^{q} \mathrm{~d} q q^{3} C(q) \times \int_{0}^{2 \pi} \mathrm{d} \phi\left|\frac{E(q v \cos (\phi))}{\left(1-v^{2}\right) \sigma_{0}}\right|^{2},
\end{gathered}
$$

where the argument of the complex modulus is the projection of the wave vector on the direction of sliding. Notice that for negative frequencies, the complex modulus becomes the complex conjugate of the modulus at positive frequencies, which permits to solve the integrals such as $\int_{0}^{2 \pi} \cdots=4 \int_{0}^{\pi / 2} \cdots$, and corresponds also to the fact that sliding in negative direction makes the same contribution as sliding in the positive direction.

3) There is a further factor $S(q)$, a correction factor which at large magnifications can be taken as $S(q) \simeq 1 / 2$, and otherwise results from some fitting calculations of the stiffness of the contact (which were done for elastic contact), as $S(q)=1 / 2+1 / 2$ $P^{2}(q)$.

All these corrections result in a final calculation which involves four nested integrals

$$
\mu \simeq \frac{1}{2} \int_{q_{0}}^{q_{1}} \mathrm{~d} q q^{3} C(q) S(q) P(q) \times \int_{0}^{2 \pi} \mathrm{d} \phi \cos (\phi) \operatorname{Im} \frac{E(q v \cos (\phi))}{\left(1-v^{2}\right) \sigma_{0}},
$$

which is not trivial to compute (at least, in our experience), and certainly does not elucidate the parametric dependences of the various branches of the friction coefficient (low, intermediate, and high velocities), as we shall attempt here.

Persson's much earlier model [8] suggested that the friction coefficient was more simply

$$
\mu \simeq C \frac{\operatorname{Im} E\left(\omega_{0}\right)}{\left|E\left(\omega_{0}\right)\right|}
$$


where $E\left(\omega_{0}\right)$ is the complex viscoelastic modulus at the frequency $\omega_{0} \sim v / l$ of the cyclic deformation at velocity $v$ of an asperity of diameter $l$. $C$ is a constant of order unity, and this form is much easier to understand as well as to recognize the crucial role of the truncation of the roughness spectrum, here in the choice if asperity diameter $l$. Adding that $\operatorname{Im} E\left(\omega_{0}\right) /\left|E\left(\omega_{0}\right)\right|$ is also of the order unity at the frequency where this ratio assumes a maximum, friction itself would be of the order unity as maximum value, as it is correct in terms of order of magnitude.

Previous fundamental contributions were made by Williams, Landel \& Ferry [9] which relate temperature and rate dependence of viscoelastic properties, interpreted by Grosch [10] which justify a single "master curve" for the temperature and velocity dependence of friction. Grosch himself had established the main results for friction of rubbery materials long ago, finding experimentally that friction could show two maxima, one attributed to adhesion with the track, where ideally should be existing even for a perfectly smooth surface [11], and the other at higher frequency due to viscoelastic losses. The present scientific and technological challenge is on how to make more "quantitative" models, following the hope that, measuring in details the viscoelastic properties of the materials, the roughness of the substrate, one could estimate friction: but there remains a few parameters to estimate, and particularly on the adhesive component, we are left ultimately with a "fitting" exercise, which at engineering level would compete with other possible alternative, including perhaps "artificial intelligence" [12].

Popov ([13], Eq. (16.12)) suggests an equation similar to Eq. (7) of Persson [8], which can be essentially attributed to a single "scale" of asperities (same diameter), which however, recognizes in factor $C$ the contribution due to the rms slope of surface $h_{r m s}^{\prime}\left(q_{1}\right)$,

$$
\mu \simeq h_{r m s}^{\prime}\left(q_{1}\right) \frac{\operatorname{Im} E\left(\omega_{0}\right)}{\left|E\left(\omega_{0}\right)\right|},
$$

and therefore clearly identifies that the friction coefficient strongly depends on the truncation of the PSD of roughness. Since this implies that $\mu \leq h_{r m s}^{\prime}\left(q_{1}\right)$ as at most we can have a peak $\operatorname{Im} E$ $\left(\omega_{0}\right) /\left|E\left(\omega_{0}\right)\right|$ close to 1 , we recognize that for this theory to be predictive, one needs to postulate a very high slope of the surface, which in turn means a measurement of roughness down to very small scales, to the order of microns in wavelength, and below. We shall see, however, that this simplified formulation is valid only in a very crude sense.

We assume Persson's theory is the most accurate analytical model presently available, although present comparison with full numerical simulations (see e.g. Scaraggi \& Persson [14]) is necessarily very limited in terms of broadness of the roughness spectrum bandwidth. Unfortunately, it involves four nested integrals which in our experience are not easy to compute numerically, and it seems important to see:

- under which conditions the "simple" formulations by early Persson or Popov (8), see also Ciavarella [15] are valid

- what the order of corrective coefficient $k$ is in a more general equation

$$
\mu \simeq k h_{r m s}^{\prime}\left(q_{1}\right) \frac{\operatorname{Im} E\left(q_{1} v\right)}{\left|E\left(q_{1} v\right)\right|}
$$

- what the main parametric dependencies are, if the dependence is not that given by this form (9) 
Here, we shall make a more detailed study, based on simple material models, like the generalized Maxwell model, or one with power law trends of the loss and storage moduli at low frequencies, to make illustrative examples.

\section{GENERALIZED MAXWELl MATERIALS}

Viscoelastic materials are typically represented with complex elastic moduli

$$
E(\omega)=\operatorname{Re} E(\omega)+i \operatorname{Im} E(\omega)=E^{\prime}(\omega)+i E^{\prime \prime}(\omega),
$$

where $E^{\prime}(\omega)$, the "storage modulus", is the material stiffness while the "loss modulus" $E^{\prime \prime}(\omega)$ is the irreversible damping: also, the ratio between loss modulus and storage modulus is dissipation factor $\tan \delta(\omega)$, which indicates the degree of viscoelasticity of a material. These factors are often determined using the Dynamic Mechanical Analysis (DMA), by a forced oscillation at a constant frequency, and interpolation and extrapolation for a wider frequency range is obtained using the time / temperature shift WLF-equation by William, Landel, Ferry [9]. Very often, a Prony series describes the complex modulus (see Fig.1), with a model comprising a parallel connection of several Maxwell elements

$$
\begin{gathered}
\operatorname{Re} E(\omega)=E_{\infty}+\sum_{i=1}^{n} E_{i} \frac{\omega^{2} \tau_{i}^{2}}{1+\omega^{2} \tau_{i}^{2}}, \\
\operatorname{Im} E(\omega)=\sum_{i=1}^{n} E_{i} \frac{\omega \tau_{i}}{1+\omega^{2} \tau_{i}^{2}},
\end{gathered}
$$

where $E_{\infty}$ is the final, equilibrium long term modulus of a tensile test (if shear modulus is measured, usually the conversion if done to Young's modulus assuming a frequencyindependent Poisson's ratio), while

$$
E_{T}=E_{\infty}+\sum_{i=1}^{n} E_{i}
$$

is the instantaneous modulus. The relaxation times are defined as $\tau_{i}=\eta_{i} / E_{i}$, where $\eta_{i}$ is the damping viscosity of the $i$-th element, and are usually ordered such that $\tau_{1}<<\tau_{2} \ldots$

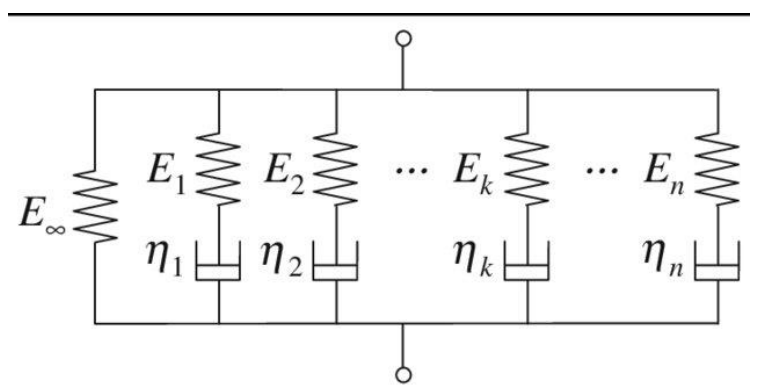

Fig. 1 A typical Prony series of a generalized Maxwell material 
There are various approaches to determine the Prony series although this is not a welldefined exercise so that often the relaxation constants are assumed, and the moduli are found by some optimization to reduce the error with experimental data [16, 17].

\subsection{A single relaxation time}

Let us consider for simplicity just one characteristic time

$$
\begin{gathered}
\operatorname{Re} E(\omega)=E_{\infty}+E_{1} \frac{\omega^{2} \tau_{1}^{2}}{1+\omega^{2} \tau_{1}^{2}}, \\
\operatorname{Im} E(\omega)=E_{1} \frac{\omega \tau_{1}}{1+\omega^{2} \tau_{1}^{2}} .
\end{gathered}
$$

We obtain from the general Persson's model (6)

$$
\mu \simeq \frac{1}{2} \int_{q_{0}}^{q} \mathrm{~d} q q^{3} C(q) S(q) P(q) \times I_{1},
$$

where

$$
\begin{aligned}
I_{1} & =\int_{0}^{2 \pi} \mathrm{d} \phi \cos (\phi) \operatorname{Im} \frac{E(q v \cos (\phi))}{\left(1-v^{2}\right) \sigma_{0}}=\frac{4}{\left(1-v^{2}\right) \sigma_{0}} \times E_{1} q v \tau_{1} \int_{0}^{\pi / 2} \mathrm{~d} \phi \frac{\cos ^{2} \phi}{1+\left(q v \tau_{1}\right)^{2} \cos ^{2} \phi} \\
& =\frac{4}{\left(1-v^{2}\right) \sigma_{0}} \times \frac{\pi E_{1}}{2 q v \tau_{1}}\left(1-\sqrt{\frac{1}{1+\left(q v \tau_{1}\right)^{2}}}\right)
\end{aligned}
$$

and

$$
G=\frac{1}{8} \int_{q_{0}}^{q} \mathrm{~d} q q^{3} C(q) \times I_{2}
$$

with

$$
I_{2}=\int_{0}^{2 \pi} \mathrm{d} \phi\left|\frac{E(q v \cos \phi)}{\left(1-v^{2}\right) \sigma_{0}}\right|^{2}=\frac{2 \pi}{\left(1-v^{2}\right)^{2} \sigma_{0}^{2}}\left[E_{\infty}^{2}+\left(E_{1}^{2}+2 E_{\infty} E_{1}\right)\left(1-\sqrt{\frac{1}{1+\left(q v \tau_{1}\right)^{2}}}\right)\right] .
$$

For a power law PSD of roughness between cutoff wave vectors $q \epsilon\left[q_{0}, q_{1}\right]$ (a choice without loss of generality if the results will turn out to depend only on the tail of the PSD which is indeed very often a power law)

$$
C(q)=C_{0} q^{-2(H+1)}
$$

for which we can give all integrals except the final one in series form involving hypergeometric functions (Mathematica can compute it, but it is here not reproduced for brevity, although some care should be used for very low velocities, where it is better to use the asymptotic expressions). It is interesting, however, to give the behavior at low and high speeds

$$
G_{\text {low } V}(q)=\frac{1}{8\left(1-v^{2}\right)^{2} \sigma_{0}^{2}} \frac{\pi C_{0}}{1-H} E_{\infty}^{2}\left(q^{2-2 H}-q_{0}^{2-2 H}\right),
$$




$$
G_{\text {high } V}(q)=\frac{1}{8\left(1-v^{2}\right)^{2} \sigma_{0}^{2}} \frac{\pi C_{0}}{1-H}\left(E_{\infty}+E_{1}\right)^{2}\left(q^{2-2 H}-q_{0}^{2-2 H}\right),
$$

which is obviously the result due to the complex modulus being essentially real at high and low frequencies, and given by $E_{\infty}$ and $E_{\infty}+E_{1}$, respectively.

Further,

$$
\begin{gathered}
I_{1, \text { low } V}(q) \simeq \frac{4}{\left(1-v^{2}\right) \sigma_{0}} \times \frac{\pi E_{1}}{2} q v \tau_{1}, \\
I_{1, \text { high } V}(q) \simeq \frac{4}{\left(1-v^{2}\right) \sigma_{0}} \times \frac{\pi E_{1}}{2 q v \tau_{1}}
\end{gathered}
$$

while

$$
\begin{gathered}
I_{2, \text { low } V}(q) \simeq \frac{4}{\left[\left(1-v^{2}\right) \sigma_{0}\right]^{2}} \times \frac{\pi E_{\infty}^{2}}{2}, \\
I_{2, \operatorname{high} V}(q) \simeq \frac{4}{\left[\left(1-v^{2}\right) \sigma_{0}\right]^{2}} \times \frac{\pi}{2}\left(E_{\infty}+E_{1}\right)^{2} .
\end{gathered}
$$

Therefore, using

$$
\operatorname{erf}\left(\frac{1}{2 \sqrt{G}}\right) \simeq \frac{1}{\sqrt{\pi G}}
$$

as can be done even for large area ratios, we obtain

$$
\begin{aligned}
\mu_{\text {lowV }}(q) & \simeq \sqrt{2 C_{0}(1-H)} \times \frac{E_{1}}{E_{\infty}} \tau_{1} v \int_{q_{0}}^{q_{1}} \mathrm{~d} q \frac{q^{2-2 H}}{\sqrt{q^{2-2 H}-q_{0}^{2-2 H}}} \\
& \simeq \sqrt{2 C_{0}(1-H)} \times \frac{E_{1}}{E_{\infty}} \tau_{1} v \frac{q_{1}^{2-H}}{2-H} \\
& \simeq \sqrt{\frac{2}{\pi}} \times\left.\frac{1-H}{2-H} h_{r m s}^{\prime}\left(q_{1}\right) \frac{\operatorname{Im} E\left(q_{1} v\right)}{\left|E\left(q_{1} v\right)\right|}\right|_{\text {low } V}
\end{aligned}
$$

where we used that rms slopes

$$
h_{r m s}^{\prime}\left(q_{1}\right)=q_{1}^{1-H} \sqrt{\frac{\pi C_{0}}{1-H}} .
$$

Therefore, notice that corrective coefficient $k$ with respect to Eq. (9) depends only on the Hurst exponent of the surface and,

$$
k \simeq \sqrt{\frac{2}{\pi}} \times \frac{1-H}{2-H}
$$

and for example $k \simeq 0.133$ for $H \simeq 0.8$. 
This confirms that the friction at low speed highly depends on $q_{1}$ as suggested originally by Persson [8], then by Popov [13] and Ciavarella [15], but also finds that the corrective coefficient can be significantly lower than one. Perhaps this is due to the nature of the single relaxation constant, which is probably a limit case. Nothing can be said $a$ priori on how far this approximation goes, however, in terms of speed range.

Therefore, it is useful to move to the other extreme at high speeds, where we find with the same procedure that

$$
\begin{gathered}
\mu_{\text {high } V}(q) \simeq \frac{\sqrt{2 C_{0}(1-H)}}{\pi} \times \frac{\pi}{\tau_{1} v} \int_{q_{0}}^{q_{1}} \mathrm{~d} q \frac{q^{-2 H}}{\sqrt{q^{2-2 H}-q_{0}^{2-2 H}}} \simeq \gamma(H) \frac{E_{1}}{E_{\infty}+E_{1}} \frac{h_{r m s}}{\tau_{1} v}, \\
\left.\Rightarrow \mu_{\text {high } V}(q) \simeq \gamma(H) \frac{\operatorname{Im} E\left(q_{1} v\right)}{\left|E\left(q_{1} v\right)\right|}\right|_{\text {high } V} q_{1} h_{r m s},
\end{gathered}
$$

where

$$
\gamma(H)=q_{0}^{H} \sqrt{\frac{2}{\pi} H(1-H)} \int_{q_{0}}^{q_{1}} \mathrm{~d} q \frac{q^{-2 H}}{\sqrt{q^{2-2 H}-q_{0}^{2-2 H}}} \simeq 0.85 \text { at } H \simeq 0.8, \text { for example. }
$$

Eq. (32) is therefore completely different in form with respect to what suggested originally by Persson [8], then by Popov [13] and Ciavarella [15], so there can be no corrective coefficient with respect to Eq. (9). In particular, as it is evident in the form (31), it does not depend on truncating large wave vector.

As an example, we introduce the case of PSD having $H=0.86, \mathrm{C}_{0}=1.152 \times 10^{-3}\left[\mathrm{~m}^{6-2 H}\right]$, and $q_{0}=10^{2.7}[1 / \mathrm{m}]$, which are realistic values for road surfaces, whereas the high truncating wave vector is initially set, according to Lorentz et al. [6], such that $h_{\text {rms }}^{\prime}=1.3$. For the material, we take $E_{\infty}=10 M P a, E_{1}=1000 M P a, \tau_{1}=7 \times 10^{-4} s$ and Fig. 2 shows the storage and loss moduli in terms of frequency.

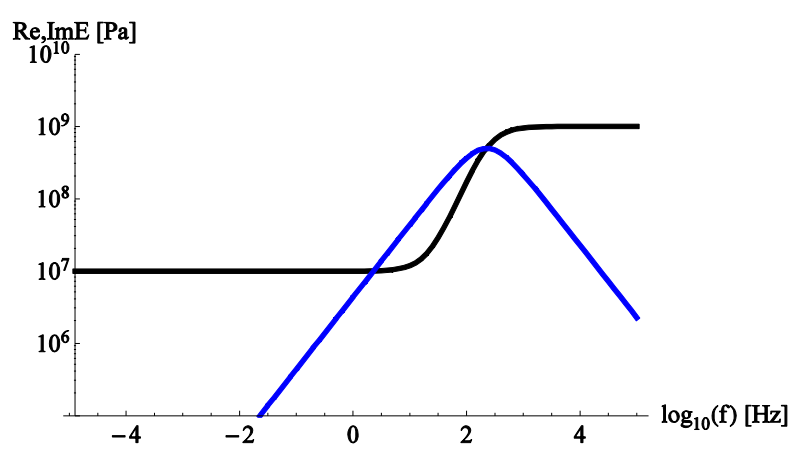

Fig. 2 An example Maxwell material with $E_{\infty}=10 \mathrm{MPa}, E_{1}=1000 \mathrm{MPa}, \tau_{1}=7 \times 10^{-4} \mathrm{~s}$. Black line indicates the storage, while blue line the loss modulus

Fig. 3 shows the results for the friction coefficient, showing that the low and high velocity approximations work quite well whereas using the full approximate (9) "simple" equation using for $k=0.098$ as in (30) does not estimate the peak friction coefficient with great accuracy, and at high speeds, it is better to use directly the high velocity approximation (31). 


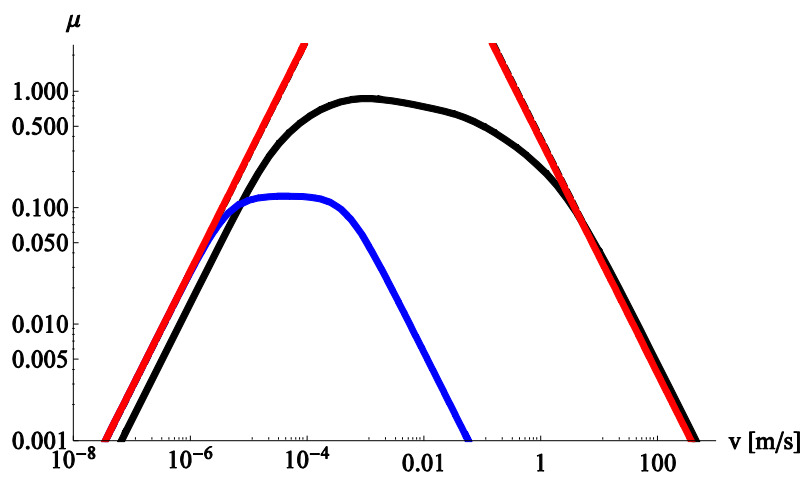

Fig. 3 Friction coefficient for the example Maxwell material of Fig. 2. Black solid line is the numerical solution of the friction coefficient with the full Persson's model, red dashed line indicate the asymptotic results (28), (31), and blue dashed line is the approximate "simple" equation (9) using for $k=0.098$ as in (30)

Fig. 4 shows the effect of varying the truncation cutoff, given this choice is arbitrary, results also in dramatic differences. In particular the solid lines indicate the full Persson's solution with $h_{\text {rms }}^{\prime}=0.69,1.3,1.8,2.1$ (black, blue, red and green lines, respectively). Notice that the high velocity branch of the friction coefficient curve does not change, as we expected from the result (31) which is instead determined by the rms amplitude of roughness, not sensitive to the truncating cutoff. It is obvious that by small changes in the choice of the truncation in $h_{\text {rms }}^{\prime}$, namely just tripling its value from 0.69 to 2.1 , we have in the low branch a change in the friction coefficient which is by orders of magnitude! Notice also that for very broad spectra of roughness, one could expect a persistently high friction coefficient for a wide range of velocities. In this sense, the "single scale" approximation (9) is increasingly poor for broad spectra, as can be expected.

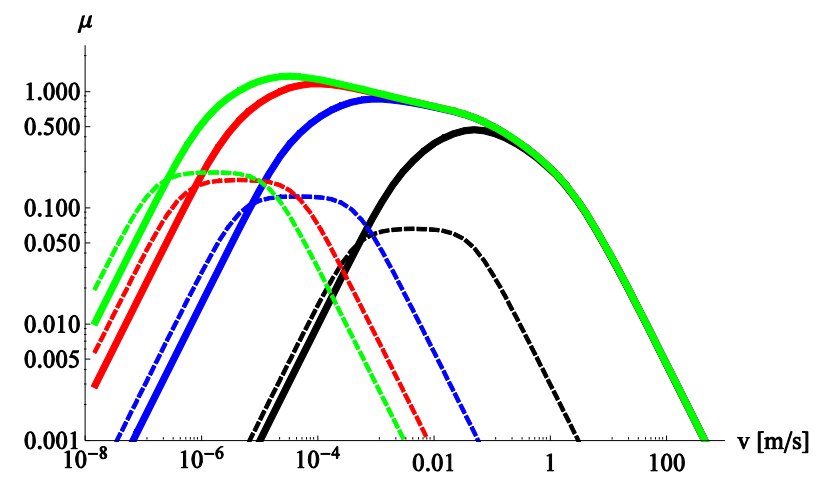

Fig. 4 Friction coefficient for the example Maxwell material of Fig. 2, while varying the upper wave vector truncation $q_{1}$ such that $h_{\text {rms }}^{\prime}=0.69,1.31 .8,2.1$ (black, blue, red and green solid lines, respectively, for the full Persson's solution). Dashed lines of the corresponding color are the approximate "simple" Eq. (9) using for $k=0.098$ as in Eq. (30) 


\subsection{Multiple relaxation times}

Let us reconsider the more general case of various relaxation times so as to cover more realistic materials. It is clear that repeating the derivation

$$
\begin{aligned}
I_{1} & =\sum_{i=1}^{n} \frac{E_{i}}{\left(1-v^{2}\right) \sigma_{0}} \int_{0}^{2 \pi} \mathrm{d} \phi \cos \phi \frac{\left(q v \tau_{i}\right)^{2} \cos ^{2} \phi}{1+\left(q v \tau_{i}\right)^{2} \cos ^{2} \phi} \\
& =\frac{4}{\left(1-v^{2}\right) \sigma_{0}} \sum_{i=1}^{n} \frac{\pi E_{i}}{2 q v \tau_{i}}\left(1-\sqrt{\frac{1}{1+\left(q v \tau_{i}\right)^{2}}}\right),
\end{aligned}
$$

so that $I_{1, \text { low } V}$ remains the same as with a single relaxation time (23), where now we replace the modulus at low or high frequency

$$
\begin{aligned}
& I_{1, \text { low } V}(q) \simeq \frac{4}{\left(1-v^{2}\right) \sigma_{0}} \times \sum_{i=1}^{n} \frac{\pi E_{i}}{2} q v \tau_{i} \simeq \frac{4}{\left(1-v^{2}\right) \sigma_{0}} \times\left.\frac{\pi}{2} \operatorname{Im} E(q v)\right|_{\text {low } \omega}, \\
& I_{1, \text { high } V}(q) \simeq \frac{4}{\left(1-v^{2}\right) \sigma_{0}} \times \sum_{i=1}^{n} \frac{\pi E_{i}}{2 q v \tau_{i}} \simeq \frac{4}{\left(1-v^{2}\right) \sigma_{0}} \times\left.\frac{\pi}{2} \operatorname{Im} E(q v)\right|_{\operatorname{high} \omega},
\end{aligned}
$$

since

$$
\left.\operatorname{Im} E(\omega)\right|_{\text {low } \omega}=\omega \sum_{i=1}^{n} \eta_{i}, \text { whereas }\left.\operatorname{Im} E(\omega)\right|_{\operatorname{high} \omega}=\frac{1}{\omega} \sum_{i=1}^{n} \frac{E_{i}}{\tau_{i}}
$$

Similarly for $I_{2}$, but we can also say

$$
\begin{gathered}
I_{2, \text { low } V}(q) \simeq \frac{2 \pi E_{\infty}^{2}}{\left[\left(1-v^{2}\right) \sigma_{0}\right]^{2}}, \\
I_{2, \text { high } V}(q)=\frac{2 \pi}{\left[\left(1-v^{2}\right) \sigma_{0}\right]^{2}}\left(E_{\infty}+\sum_{i=1}^{n} E_{i}\right)^{2} .
\end{gathered}
$$

We obtain from the general Persson's model (6) (and again for a power law PSD of roughness), a similar behavior at low and high speeds

$$
\begin{gathered}
G_{\mathrm{low} V}(q)=\frac{1}{8\left(1-v^{2}\right)^{2} \sigma_{0}^{2}} \frac{\pi C_{0}}{1-H} E_{\infty}^{2}\left(q^{2-2 H}-q_{0}^{2-2 H}\right) \\
G_{\mathrm{high} V}(q)=\frac{1}{8\left(1-v^{2}\right)^{2} \sigma_{0}^{2}} \frac{\pi C_{0}}{1-H}\left(E_{\infty}+\sum_{i=1}^{n} E_{i}\right)^{2}\left(q^{2-2 H}-q_{0}^{2-2 H}\right) .
\end{gathered}
$$

Therefore, using Eq. (27), we obtain the same equations (28) and (32) as for the single relaxation time. This seems to suggest that these results should have greater generality. However, in many cases, experimentally we observe that loss and storage modulus have power law form in frequency for many compounds of interest, as we shall discuss in the next paragraph. 


\section{POWER LAW Form OF THE LOSS AND STORAGE MOdUluS}

It appears that in the practical range of interest for rubber compounds used in tires (see Lorenz et al. [6]), we observe storage and loss moduli which seem to follow power laws (see Fig. 5 for three distinct examples). Let us therefore consider this case, which permits also a simple estimate of the relevant equations in closed form at low speeds.
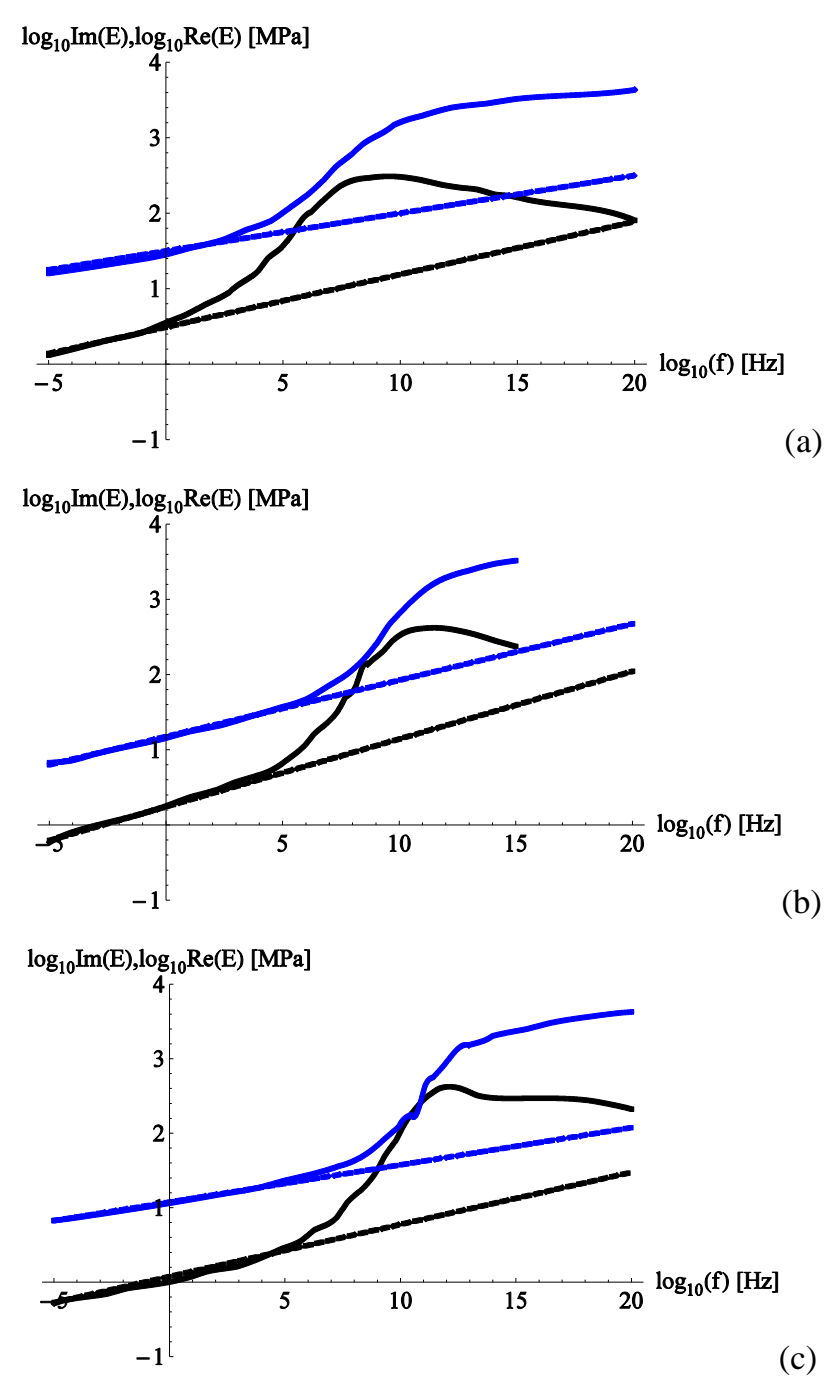

(b)

(c)

Fig. 5 Real (solid blue) and Imaginary (solid black) parts of the viscoelastic modulus in Lorenz et al. [6] rubber compound A, B, C (respectively in Fig. 5 a,b,c) together with power law approximations (dashed lines) at low frequencies. These are $E=10^{1.5} f^{0.05}+$ $i 10^{0.49} f^{0.07}, E=10^{1.175} f^{0.075}+i 10^{0.243} f^{0.09}, E=10^{1.175} f^{0.05}+i 10^{0.075} f^{0.07}$, for A, B, C, respectively 
Let us consider the following low-frequency end approximation of power law behavior,

$$
\operatorname{Re} E(\omega)=10^{\alpha_{r}} \omega^{\beta_{r}} \text { and } \operatorname{Im} E(\omega)=10^{\alpha_{i}} \omega^{\beta_{i}}
$$

where for example Lorenz et al. [6] rubber compound A, B, C suggest that $\beta_{\mathrm{r}} \simeq 0.05-$ 0.075 whereas $\beta_{i} \simeq 0.07-0.09$. This seems to suggest that while for the real part, we are not too far from the constant values which in principle we expect at very low frequencies for a (even generalized) Maxwell material, the imaginary part is certainly remote from the single relaxation constant material, which would have $\beta_{i}=1$.

Now, with the same notation as for the Maxwell materials, we obtain the integrals

$$
I_{1}=\frac{4}{\left(1-v^{2}\right) \sigma_{0}} \times 10^{\alpha_{i}}(q v)^{\beta_{i}} \frac{\sqrt{\pi}}{2} \frac{\Gamma\left(1+\beta_{i} / 2\right)}{\Gamma\left(3 / 2+\beta_{i} / 2\right)}
$$

and

$$
\begin{aligned}
I_{2} & =\frac{4}{\left[\left(1-v^{2}\right) \sigma_{0}\right]^{2}} \int_{0}^{\pi / 2} \mathrm{~d} \phi\left|10^{\alpha_{r}}(q v \cos \phi)^{\beta_{r}}\right|^{2} \\
& \simeq \frac{4}{\left[\left(1-v^{2}\right) \sigma_{0}\right]^{2}} \times 10^{2 \alpha_{r}}(q v)^{2 \beta_{r}} \frac{\sqrt{\pi}}{2} \frac{\Gamma\left(1 / 2+\beta_{r}\right)}{\Gamma\left(1+\beta_{r}\right)},
\end{aligned}
$$

where we have considered that at low $v$ the real part of the modulus dominates over the imaginary.

Also,

$$
G=\frac{1}{8} \frac{4}{\left[\left(1-v^{2}\right) \sigma_{0}\right]^{2}} \times 10^{2 \alpha_{r}} v^{2 \beta_{r}} \frac{\sqrt{\pi}}{2} \frac{\Gamma\left(1 / 2+\beta_{r}\right)}{\Gamma\left(1+\beta_{r}\right)} C_{0} \frac{q^{2\left(\beta_{r}+1-H\right)}-q_{0}^{2\left(\beta_{r}+1-H\right)}}{2\left(\beta_{r}+1-H\right)}
$$

Therefore, using Eq. (27),

$$
\begin{aligned}
\mu & \simeq \sqrt{\pi C_{0}} \frac{10^{\alpha_{i}-\alpha_{r}} v^{\beta_{i}-\beta_{r}} \frac{\Gamma\left(1+\beta_{i} / 2\right)}{\Gamma\left(3 / 2+\beta_{i} / 2\right)}}{\sqrt{\frac{\Gamma\left(1 / 2+\beta_{r}\right)}{\Gamma\left(1+\beta_{r}\right)} \frac{\pi \sqrt{\pi}}{2\left(\beta_{r}+1-H\right)}} \int_{q_{0}}^{q_{1}} \mathrm{~d} q \frac{q^{1-2 H+\beta_{i}}}{\sqrt{q^{2\left(\beta_{r}+1-H\right)}-q_{0}^{2\left(\beta_{r}+1-H\right)}}}} \\
& \left.\simeq \frac{\sqrt{1-H}}{1-H+\beta_{i}-\beta_{r}} \frac{\frac{\Gamma\left(1+\beta_{i} / 2\right)}{\Gamma\left(3 / 2+\beta_{i} / 2\right)}}{\sqrt{\frac{\Gamma\left(1 / 2+\beta_{r}\right)}{\Gamma\left(1+\beta_{r}\right)} \frac{\pi \sqrt{\pi}}{2\left(\beta_{r}+1-H\right)}}} h_{r m s}^{\prime}\left(q_{1}\right) \frac{\operatorname{Im} E\left(q_{1} v\right)}{\left|E\left(q_{1} v\right)\right|}\right|_{\text {low } V}
\end{aligned}
$$

since

$$
\left.\frac{\operatorname{Im} E\left(q_{1} v\right)}{\left|E\left(q_{1} v\right)\right|}\right|_{\text {low } V} \simeq 10^{\alpha_{i}-\alpha_{r}}\left(q_{1} v\right)^{\beta_{i}-\beta_{r}}
$$

Eq. (46) is an equation of the form suggested originally by Persson [8], then by Popov [13] and Ciavarella [15], but where the corrective coefficient as in Eq. (9) is given by 


$$
k=\frac{\sqrt{1-H}}{1-H+\beta_{i}-\beta_{r}} \frac{\frac{\Gamma\left(1+\beta_{i} / 2\right)}{\Gamma\left(3 / 2+\beta_{i} / 2\right)}}{\sqrt{\frac{\Gamma\left(1 / 2+\beta_{r}\right)}{\Gamma\left(1+\beta_{r}\right)} \frac{\pi \sqrt{\pi}}{2\left(\beta_{r}+1-H\right)}}},
$$

which in particular we find that for the Maxwell material having $\beta_{r}=0$ and $\beta_{i}=1$, we return to Eq. (30). Notice therefore from Eq. (48), that the actual behavior at low speeds changes significantly the coefficients $k$ needed in the simple Eq. (9) to estimate the ascending branch of the friction curve.

Fig. 6 compares some example coefficients $k$ as in Eq. (48) as a function of $\beta_{i}$ in the range from $10^{-3}$ to 1 , for a few cases of $\beta_{r}=0,0.05,0.1,0.15$ (black, blue, red, green curves, respectively). It is evident that $k$ can vary significantly both below and above 1 , tends to be independent on $\beta_{i}$ at low $\beta_{i}$. However, it is quite close to 1 for the typical materials shown in Lorenz et al. [6] rubber compound A,B,C, which explains why there appeared to be even too success with the simple equation in a previous paper (Ciavarella [15]).

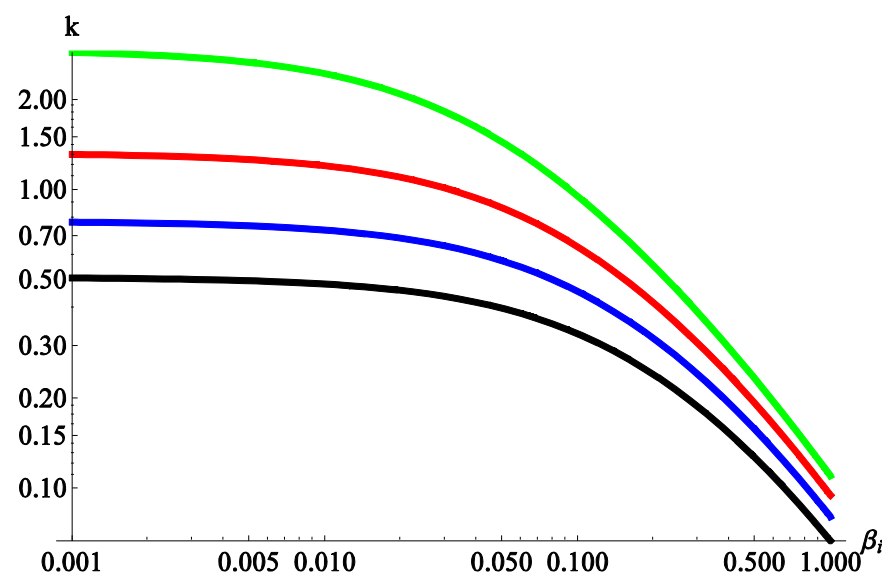

Fig. 6 The multiplicative coefficient $\mathrm{k}$ as in Eq. (48) for using the approximate "simple" Eq. (9) at low velocities, for generic power law approximations of the storage and loss moduli as a function of $\beta_{i}$ in the range from 0 to 1 , for a few cases of $\beta_{r}=0$, $0.05,0.1,0.15$ (black, blue, red, green curves, respectively)

\section{DISCUSSION}

Recently [18], some actual data from Tolpekina and Persson [19] have been analyzed using the simplified formulation, and adhesive contribution. It has been remarked that the adhesive contribution seems more important than the viscoelastic one, perhaps more important also than what expected, for example, from earlier studies of Grosch. Unfortunately, the adhesive contribution fundamentally to date is modeled with empirical fitting equations, which have a "bell-shape" which is quite similar to the viscoelastic friction contribution, in large parts of the velocity spectrum. Hence, it results that it is quite difficult to estimate with 
precision the relative contribution since various choices of the truncation wave vector, and other fundamentally arbitrary constants, result in equally satisfactory fitting of the experimental data. Additionally, viscoelastic materials nonlinear effects seem to strongly affect the adhesive models (and strictly speaking also the viscoelastic ones, although it is not clear if Tolpekina and Persson [19] consider this), and their role depends also crucially on the choice of the truncating wavelength of roughness. Hence, while we have made a significant progress in the mathematical solution of the viscoelastic contribution in highly idealized conditions, this seems very remote from practical "predictive" capabilities, and the complexities of the full multiscale theories do not help in clarifying the subject for a large audience. What is needed is, instead, that more researchers work actively in this field by comparing theories and results, and ideally, collecting "Round Robin" results and benchmark cases. Our effort here is a small attempt in this direction.

\section{CONCLUSIONS}

We have derived some simple results for the fully multiscale Persson's theory as applied to quite important materials models, like generalized Maxwell, or with power-law storage and loss modulus at low frequencies. This has permitted to elucidate that the friction coefficient at low velocity of sliding, where we expect most contacts operate, show a simple dependency on the ratio of imaginary and absolute value of the modulus and on the rms slope of the profile noticed earlier by Persson, Popov and also the present author, which also implies a very high sensitivity to the choice of the truncating wave vector of roughness. However, we also find that this "simple equation" has a multiplicative coefficient which depends on the form of the viscoelastic modulus, and we have given approximate but simple closed form results for simple cases. The peak of the friction coefficient, however, cannot be estimated accurately with the simple equation. At high velocities, it is found that the descending (unstable) branch at high speed mainly on the amplitude of roughness, and, therefore, would not depend on the choice of the truncating wave vector. There remain great uncertainties in "predicting" friction coefficient since orders of magnitude variation can be found changing the truncating wave vector.

Acknowledgements: A.P. is thankful to the DFG (German Research Foundation) for funding the projects HO 3852/11-1 and PA 3303/1-1.

\section{REFERENCES}

1. Ciavarella, M., Demelio, G., Barber, J.R., Jang, Y.H., 2000, Linear elastic contact of the Weierstrass profile, Proceedings of the Royal Society of London, Series A: Mathematical, Physical and Engineering Sciences, 456(1994), pp. 387-405.

2. Persson, B.N.J., 2001, Theory of rubber friction and contact mechanics, The Journal of Chemical Physics, 115(8), pp. 3840-3861.

3. Persson, B.N.J., Albohr, O., Tartaglino, U., Volokitin, A.I., Tosatti, E., 2005, On the nature of surface roughness with application to contact mechanics, sealing, rubber friction and adhesion, Journal of physics: Condensed matter, 17(1), pp. 1-62.

4. Greenwood, J.A., Williamson, J.B.P., 1966, Contact of nominally flat surfaces, Proceedings of the royal society of London, Series A, Mathematical and physical sciences, 295(1442), pp 300-319. 
5. Putignano, C., Afferrante, L., Carbone, G., Demelio, G., 2012, A new efficient numerical method for contact mechanics of rough surfaces, International Journal of Solids and Structures, 49(2), pp. 338-343.

6. Lorenz, B., Oh, Y.R., Nam, S.K., Jeon, S.H., Persson, B.N.J., 2015, Rubber friction on road surfaces: Experiment and theory for low sliding speeds, The Journal of chemical physics, 142(19), p. 194701.

7. Carbone, G., Putignano, C., 2014, Rough viscoelastic sliding contact: theory and experiments, Physical Review E, 89(3), p. 032408.

8. Persson, B.N.J., 1998, On the theory of rubber friction, Surface Science, 401(3), pp. 445-454.

9. Williams, M.L., Landel, R.F., Ferry, J.D., 1955, The temperature dependence of relaxation mechanisms in amorphous polymers and other glass-forming liquids, Journal of the American Chemical society, 77(14), pp. 701-3707.

10. Grosch, K.A., 1963, The relation between the friction and visco-elastic properties of rubber, Proceedings of the Royal Society of London, Series A, Mathematical and Physical Sciences, 274(1356), pp. 21-39.

11. Grosch, K.A., 1996, The rolling resistance, wear and traction properties of tread compounds, Rubber Chemistry and technology, 69(3), pp. 495-568.

12. Khaleghian, S., Emami, A., Taheri, S., 2017, A technical survey on tire-road friction estimation, Friction, 5(2), pp. 123-146.

13. Popov, V.L., 2010, Contact mechanics and friction: physical principles and applications, Springer Science \& Business Media, Berlin, Heidelberg.

14. Scaraggi, M., Persson, B.N.J., 2015, Friction and universal contact area law for randomly rough viscoelastic contacts, Journal of Physics: Condensed Matter, 27(10), p. 105102.

15. Ciavarella, M., 2018, A simplified version of Persson's multiscale theory for rubber friction due to viscoelastic losses, Journal of Tribology, 140(1), p. 011403.

16. https://it.mathworks.com/matlabcentral/fileexchange/68710-dma2prony_opt (last access: 10.05.2019)

17. Jalocha, D., Constantinescu, A., Neviere, R., 2015, Revisiting the identification of generalized Maxwell models from experimental results, International Journal of Solids and Structures, 67, pp. 169-181.

18. Genovese, A., Carputo, F., Ciavarella, M., Farroni, F., Papangelo, A., Sakhnevych, A., 2019, Analysis of multiscale theories for viscoelastic rubber friction, Proceedings AIMETA 2019 XXIV Conference The Italian Association of Theoretical and Applied Mechanics Rome, Italy, 15-19 September.

19. Tolpekina T.V., Persson B.N.J., 2019, Adhesion and friction for three tire tread compounds, Lubricants, $7(3)$, p. 20. 